\title{
The Efficacy of a Transurethral Incision for Diurnal and Nocturnal Enuresis in Young Males
}

\author{
Shohei Tobu Mitsuru Noguchi Kohei Takahara Yuka Ichibagase \\ Saya Ikoma Kazuma Udo Maki Nanri Jiro Uozumi \\ Department of Urology, Saga University, Saga, Japan
}

\section{Key Words}

Pediatric case • Surgical treatment - Urinary incontinence

\begin{abstract}
Introduction: In this study, we investigated the effects of treatment with a transurethral incision (TUI) for congenital urethral stenosis, which was accompanied by diurnal and nocturnal enuresis. Methods: We recruited 21 young males who presented to our department for the treatment of diurnal and nocturnal enuresis from January 2010 to March 2014. All patients underwent TUI due to urethral stricture found by a close investigation. We surveyed each case to evaluate the improvement of diurnal and/or nocturnal enuresis after TUI. Results: One and a half years after TUI, an improvement in diurnal enuresis was observed in 17 of 21 cases (80.9\%), whereas that of nocturnal enuresis was observe in only 7 of 21 cases (33.3\%), showing the significant contribution of TUI to the improvement of diurnal enuresis ( $p=0.001$ ). In the case of diurnal enuresis, continual improvement was observed more than a year after surgery, whereas no improvement was observed in nocturnal enuresis at more than 6 months after surgery. Conclusion: TUI is more effective for diurnal enuresis than nocturnal enuresis. At postoperative 6 months, clinicians should thus consider other etiologies for unresponsive cases and start other treatment options.
\end{abstract}

\section{KARGER}

Fax +4161306 1234

E-Mail karger@karger.ch

www.karger.com
(C) 2015 S. Karger AG, Basel

1015-9770/14/0092-0079\$38.00/0

Accessible online at:

www.karger.com/cur

\section{Introduction}

Diurnal and nocturnal enuresis in young males is not rare. Enuresis is defined as involuntary urination of children over 5 years of age. Generally, most children stop diurnal enuresis at 4 years of age. The authoritative paper in this field reported that the annual spontaneous cure rate was $14 \%$ between 5 and 9 years of age, and was $16 \%$ between 10 and 19 years of age. Thirty-three patients (3\%) continued to experience enuresis after 20 years of age [1]. Another previous report demonstrated that $15 \%$ of 5-year-old children experience enuresis; $75 \%$ of them demonstrated only nocturnal enuresis, $10 \%$ demonstrated only diurnal enuresis, and $15 \%$ demonstrated both types of enuresis.

Children with a lower urinary tract obstruction might display coexisting diurnal and nocturnal symptoms and pollakisuria [2, 3]. Transurethral incision (TUI) is a common procedure for urethral stricture. The efficacy of this procedure for dysuria by urethral stricture has been demonstrated by many reports [4].

We occasionally experience each type of enuresis in young males who are successfully treated by TUI. However, the efficacy of TUI for young males with urethral stenosis has not yet been adequately discussed because nocturnal enuresis is often improved by observation and is not lethal. However, nocturnal enuresis with diurnal enuresis is often intractable. Moreover, differences in the efficacy of TUI between diurnal and nocturnal enuresis have not been sufficiently discussed.

Shohei Tobu

Department of Urology, Saga University

5-1-1 Nabeshima Saga City

JP-840-0832, Saga (Japan)

E-Mail toubu7@hotmail.com 
Table 1. Patient characteristics

\begin{tabular}{ll}
\hline $\begin{array}{l}\text { Patient characteristics } \\
(\mathrm{n}=21)\end{array}$ & Value \\
\hline Age (years) & $7.2(5-10)^{*}$ \\
$\begin{array}{l}\text { Uroflowmetric pattern } \\
\text { Bell shape }\end{array}$ & $1 / 21(4.7 \%)$ \\
$\quad$ Staccato type & $2 / 21(9.5 \%)$ \\
$\quad$ Tower type & $1 / 21(4.7 \%)$ \\
Interrupted type & $2 / 21(9.5 \%)$ \\
$\quad$ Plateau type & $15 / 21(71.4 \%)$ \\
Location of urethral stenosis & \\
$\quad$ Anterior & $2 / 21(9.5 \%)$ \\
Posterior & $19 / 21(90.5 \%)$
\end{tabular}

*Median (range)

In this study, we investigated the effects of treatment with TUI for congenital urethral stricture, which was accompanied by diurnal and nocturnal enuresis.

\section{Materials and Methods}

Of the children who presented to our department for the treatment of diurnal and nocturnal enuresis from January 2010 to March 2014, 21 males who underwent TUI due to urethral stricture found by a close investigation for each type of enuresis were included in the study. We excluded cases that had vesicoureteral reflux or a neurogenic bladder (Table 1).

The patients' ages ranged from 5 to 10 years of age (mean age 7.2 years). There were no drop-outs during the study period. All patients were followed-up for at least 1.5 years.

We surveyed each case to evaluate the improvement of diurnal and nocturnal enuresis after TUI, including the length of time it took to achieve an improvement after surgery. We defined an improvement as less than two episodes per month of nocturnal enuresis and/or diurnal enuresis. Informed written consent was obtained from the guardians of all patients. All statistical analyses were carried out on a personal computer using the Ekuseru-Toukei 2010 statistical software package (SSRI, Tokyo, Japan). The chi-square test and Kaplan-Meier method were used to assess the efficacy of TUI for each type of enuresis. Statistical significance was considered to exist at a value of $\mathrm{p}<0.05$.

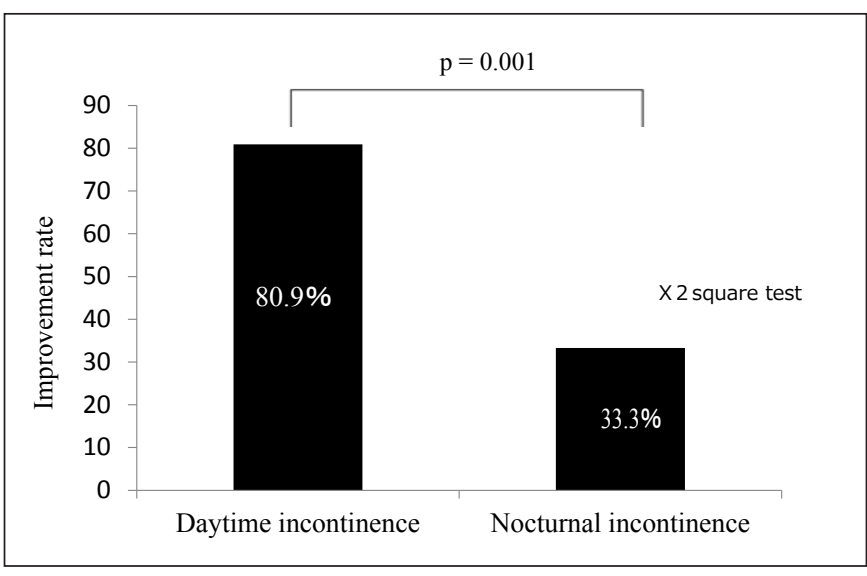

Fig. 1. TUI was more effective for daytime incontinence than for nocturnal incontinence.

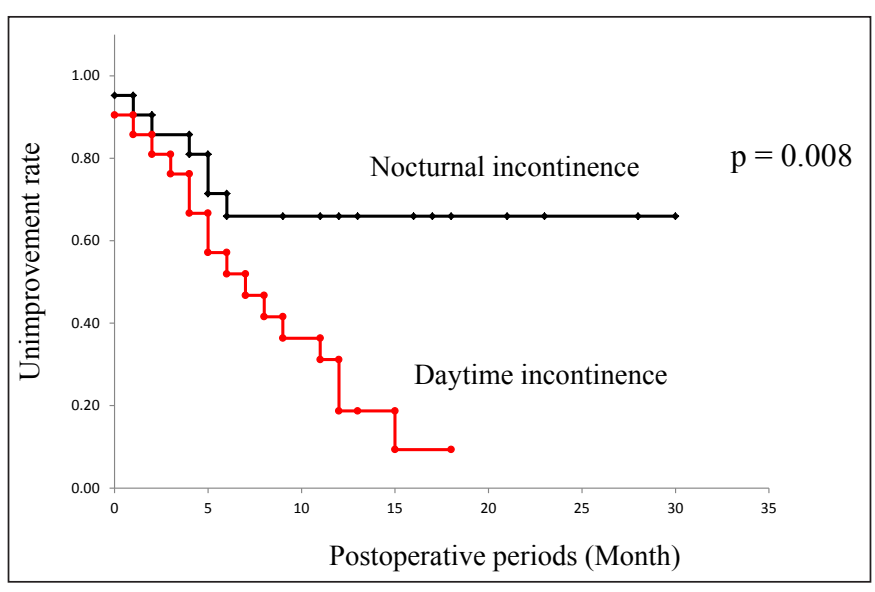

Fig. 2. Daytime incontinence improved within a shorter time period postoperatively than nocturnal incontinence.

\section{Results}

One and a half years after TUI, an improvement in diurnal enuresis was observed in 17 of 21 cases (80.9\%), whereas that of nocturnal enuresis was observed in only 7 of 21 cases $(33.3 \%)$, showing the significant contribution of TUI to the improvement of diurnal enuresis ( $\mathrm{p}=$ 0.001 , fig. 1 ).

In the case of diurnal enuresis, continual improvement was observed more than a year after surgery, whereas no improvement was observed in nocturnal enuresis at more than six months after surgery (fig. 2). 


\section{Discussion}

Nakamura et al. [5] previously reported that the improvement rates after TUI for nocturnal and diurnal enuresis were $30 \%$ and $50 \%$, respectively, at postoperative 6 months. Moreover, dyssynergia could change the improvement rate, and $35 \%$ of these patients had dyssynergia.

Our study demonstrated a difference in the improvement rate of TUI between nocturnal and diurnal enuresis using the Kaplan-Meier estimate, although our sample size $(n=21)$ was small. However, our results suggest that the etiology of diurnal enuresis might be different from that of nocturnal enuresis. TUI could effectively improve diurnal enuresis, but not nocturnal enuresis.
Interestingly, our data demonstrated that nocturnal enuresis is less likely to improve within six months after surgery, indicating the necessity of considering whether to initiate other treatments, such as drug therapy or alarm therapy, in unresponsive cases. Since diurnal enuresis often triggers the problem of bullying more frequently than nocturnal enuresis, prompt treatment for diurnal enuresis is required. As TUI is expected to lead to more effective responses for diurnal enuresis, it should be applied in cases that are considered to be caused by urethral stricture.

In conclusion, TUI is more effective for diurnal enuresis than nocturnal enuresis. At postoperative 6 months, clinicians should thus consider other etiologies for unresponsive cases and start other treatment options.

\section{References}

1 Forsythe WI, Redmond A: Enuresis and spontaneous cure rate: study of 1129 enuretis. Arch Dis Child 1974;49:259-263.

2 Cutler C, Middleton AW Jr, Nixon GW: Radiographic findings in children surveyed for enuresis. Urology 1978;11:480-482.
3 Kawauchi A, Kitamori T, Imada N, Tanaka Y, Watanabe H: Urological abnormalities in 1,328 patients with nocturnal enuresis. Eur Urol 1996;29:231-234.

4 Kajbafzadeh AM, Elmi A, Payabvash S, Sina A, Arshadi H, Sadeghi Z: The urethral valve of Guérin and lacuna magna: clinical presentations and urodynamic findings. Pediatr Surg Int 2007;23:873-878.
5 Nakamura S, Kawai S, Kubo T, Kihara T, Mori K, Nakai H: Transurethral incision of congenital obstructive lesions in the posterior urethra in boys and its effect on urinary incontinence and urodynamic study. BJU Int 2011;107:1304-1311. 